a linear system approach. Such approach has not been successful in explaining and/or predicting RRIs satisfactorily.

Objectives To develop and validate an artificial intelligence (AI) algorithm in order to identify RRI risk profiles in recreational runners.

Design Mathematical model.

Settings São Paulo, Brazil.

Participants 191 recreational runners.

Assessment of Risk Factors This was a 3-step AI study using data from a prospective cohort study. In step 1, variable selection and exploratory analyses were conducted in the original $(n=191)$ and simulated data $(n=5000)$. In step 2 , the AI algorithm was developed using self-organising maps, k-means and probabilistic neural networks. The algorithm was trained in $80 \%(n=4000)$ of the simulated data, and validated using the remaining $20 \%(n=1000)$. Characterisation of RRI risk profiles was performed in step 3 .

Main Outcome Measures RRI risk profiles were established based on the groups created by the developed algorithm. Descriptive analyses were performed to summarise the risk profiles.

Results The variables with greatest influence in the algorithm were: sex; running intensity; history of RRIs; and current musculoskeletal discomfort related to running. Five groups were suggested by the algorithm. Male runners reporting previous RRIs and running in low-to-moderate intensities ( $>6$ $\mathrm{min} / \mathrm{km}$ ) were at the highest risk of RRIs. Male runners reporting previous RRIs and running in high intensities (3 to $5 \mathrm{~min} / \mathrm{km}$ ) in about $32.1 \%$ of the time were at the lowest risk of RRIs. The classification accuracy of the algorithm presented a median of $99.6 \%$ (interquartile range: $99.5 \%$ to 99.8\%).

Conclusions A non-linear system approach using AI and machine learning techniques were successful in developing an RRI risk profile algorithm for recreational runners.

\section{ENVIRONMENTAL FACTORS, TRAINING FACTORS, RACE PACE AND DISTANCE, OLDER AGE, AND METABOLIC/ ENDOCRINE DISEASE ARE INDEPENDENT RISK FACTORS ASSOCIATED WITH ADVERSE EVENTS IN RUNNERS: A SAFER STUDY IN 76654 STARTERS}

\begin{abstract}
1,2,3 Martin Schwellnus, ${ }^{1}$ Nicola Sewry, ${ }^{4,5,6}$ Mats Borjesson, ${ }^{7}$ Sonja Swanevelder, 7,8 Esme Jordaan. 'Sport, Exercise Medicine and Lifestyle Institute (SEMLI), Pretoria, South Africa; ${ }^{2}$ IOC Research Centre, South Africa, Pretoria, South Africa; ${ }^{3}$ Emeritus Professor of Sport and Exercise Medicine, Faculty of Health Sciences, University of Cape Town, South Africa, Cape Town, South Africa; ${ }^{4}$ Institute of Neuroscience and Physiology, Sahlgrenska Academy, Göteborg University, Göteborg, Sweden; ${ }^{5}$ Center for Health and Performance, Göteborg University, Göteborg, Sweden; ${ }^{6}$ Sahlgrenska University Hospital/Östra, Göteborg, Göteborg, Sweden; ${ }^{7}$ Biostatistics Unit, South African Medical Research Council, Cape Town, South Africa; ${ }^{8}$ Statistics and Population Studies Department, University of the Western Cape, Cape Town, South Africa
\end{abstract}

\subsection{6/bjsports-2021-IOC.64}

Background Pre-race medical screening at endurance events has recently been suggested, but there are limited data on the use of pre-race questionnaire data to predict adverse events (defined as medical encounters or not finishing a race).

Objective To determine if risk factors, that are identified from a pre-race medical screening questionnaire, are associated with adverse events during a distance running event.

Design Prospective study, with cross-sectional analyses.
Setting 2012-2015 Two Oceans marathon races $(21.1 \mathrm{~km}, 56$ $\mathrm{km})$, South Africa.

Patients (or Participants) 76654 race entrants $(21.1 \mathrm{~km}$ and 56 $\mathrm{km}$ race).

Interventions (or Assessment of Risk Factors) Upon entering the race, all entrants completed a pre-race medical screening questionnaire. Based on the responses to the questionnaire, runners received a targeted educational intervention to reduce medical encounters (ME). All individual race day data was collected, and this included AE data as follows: runners that did-not-finish (DNF) the race (collected from the race organisers), and runners who had a medical encounter (ME) (collected by race day medical staff).

Main Outcome Measurements Using a multiple regression model, we report the prevalence ratio (PR: 95\% CI) of risk factors associated with the adverse event rate (AE) (defined as DNF or ME), using race day data and individual pre-race medical screening data.

Results Independent risk factors associated with $\mathrm{AE}$ in the Two Oceans Races were: males >45years (1.2: 1.0-1.3; $\mathrm{p}=0.0210)$, females >55years (1.5: 1.1-2.1; $\mathrm{p}=0.0051)$, slower training pace (increase of $1 \mathrm{~min} / \mathrm{km}, 1.2$ : 1.1-1.2; $\mathrm{p}<0.0001$ ), increased training frequency per week (increase of 2 times/week, 1.2: 1.1-1.3; $\mathrm{p}=0.0017)$, and a history of metabolic/endocrine disease (1.6: 1.2-2.1; $\mathrm{p}=0.0006$ ).

Conclusions Environmental and training factors, older age, race pace and distance, and history of metabolic/endocrine disease were independent risk factors associated with adverse events in a mass-participation distance running event. These factors could be considered when developing and implementing pre-race education material that is disseminated to participants prior to the event.

\section{SELF-REPORTED MEDICAL CONDITIONS/MEDICATION USE, OLDER FEMALES, AND SLOW PACE ARE INDEPENDENT RISK FACTORS FOR ALL MEDICAL ENCOUNTERS IN A 90 KM ULTRAMARATHON: A SAFER STUDY IN 70328 RUNNERS OVER 5 YEARS}

${ }^{1,2}$ Nicola Sewry, ${ }^{3}$ Jeremy Boulter, 1,2Martin Schwellnus. ${ }^{1}$ Sport, Exercise Medicine and Lifestyle Institute (SEMLI), Pretoria, South Africa; ${ }^{2}$ IOC Research Centre, South Africa, Pretoria, South Africa; ${ }^{3}$ Medical Director, Comrades Marathon, Pietermaritzburg, South Africa

\subsection{6/bjsports-2021-IOC.65}

Background There is a high incidence of medical encounters (MEs) in ultramarathon road running races but risk factors associated with MEs in events have not been well investigated Objective To determine the independent risk factors for all medical encounters in a $90 \mathrm{~km}$ ultramarathon running race.

Design Retrospective clinical audit, cross-sectional analysis

Setting 2014-2018 Comrades ultramarathon $(90 \mathrm{~km}$, alternating annually between an 'up' vs. a 'down' run between a coastal and inland city), South Africa

Participants 70328 race starters

Interventions All participants voluntarily completed two medical-related questions (history of allergies and/or a medical condition/or use medication) upon entry for the event. Race day data, including 'up' vs. 'down' run, start time, finish time, age and sex of starters, was recorded by the race organisers. MEs were recorded by medical staff during and immediately after the event. 